International Journal of Pure and Applied Mathematics

Volume 85 No. 6 2013, 1075-1086

ISSN: 1311-8080 (printed version); ISSN: 1314-3395 (on-line version)

url: http://www.ijpam.eu

doi: http://dx.doi.org/10.12732/ijpam.v85i6.11

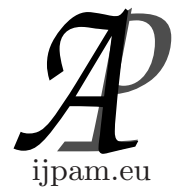

\title{
STRONG INVARIANT APPROXIMATION PROPERTY FOR DISCRETE GROUPS
}

\author{
Kankeyanathan Kannan \\ Department of Mathematics and Statistics \\ University of Jaffna \\ SRI LANKA
}

\begin{abstract}
Let $G$ be a countable exact discrete group. We show that $G$ has the approximation property if and only if

$$
C_{u}^{*}(G, S)^{G}=C_{\lambda}^{*}(G) \otimes S
$$

for any Hilbert space $\mathcal{H}$ and closed subspace $S \subseteq \mathcal{H}$, we have where $C_{u}^{*}(G)$ is the uniform Roe algebra. This answers a question of J. Zacharias.

AMS Subject Classification: 20F65, 18G60

Key Words: strong invariant approximation property, uniform Roe algebras, invariant approximation property
\end{abstract}

\section{Introduction}

The purpose of this paper is to provide an illustration of an interesting and nontrivial interaction between analytic and geometric properties of a group. We provide approximation property of operator algebras associated with discrete groups. There are various notions of finite dimensional approximation properties for $C^{*}$ - algebras and more generally operator algebras. Some of these (approximation properties) notations will be defined in this paper, the reader is referred to [2], [3] and [8] for these a beautiful concept: Haagerup discovery that that the reduced $C^{*}-$ algebra $\mathbf{F}_{n}$ has the metric approximation property, Higson and Kasparov's resolution of the Baum-connes conjecture for the Haagerup groups. We studies analytic techniques from operator theory that encapsulate geometric properties of a group. On approximation properties

Received: June 2, 2013

(c) 2013 Academic Publications, Ltd. url: www.acadpubl.eu 
of group $C^{*}$ - algebras is everywhere; it is powerful, important, backbone of countless breakthroughs.

Roe considered the discrete group of the reduced group $C^{*}-$ algebra of $C_{r}^{*}(G)$ is the fixed point algebra $\{A d \rho(t): t \in G\}$ acting on the uniform Roe algebra $C_{u}^{*}(G)[8]$. A discrete group $G$ has natural coarse structure which allows us to define the the uniform Roe algebra, $C_{u}^{*}(G)[8]$. We say that the uniform Roe algebra, $C_{u}^{*}(G)$, is the $C^{*}$ - algebra completion of the algebra of bounded operators on $\ell^{2}(X)$ which have finite propagation. The reduced $C^{*}-$ algebra $C_{r}^{*}(G)$ is naturally contained in $C_{u}^{*}(G)$ [8]. According to [Roe] [8] $G$ has the invariant approximation property (IAP) if

$$
C_{\lambda}^{*}(G)=C_{u}^{*}(G)^{G} .
$$

We give a general exposition of invariant approximation property(IAP), which was initiated by Roe [8].

Theorem 1.1. For a discrete exact group $G$. $G$ has the strong invariant approximation property if for any closed subspace $S \subseteq \mathbb{B}(H)$ the equality

$$
C_{u}^{*}(G, S)^{G}=C_{\lambda}^{*}(G) \otimes S \text { holds. }
$$

Proposition 1.2. If $G=S L_{2}(\mathbb{Z})$. Then there exist any Hilbert space $\mathbb{H}$ and closed subspace $S \subseteq H$ such that property if and only if

$$
C_{u}^{*}(G, S)^{G} \neq C_{\lambda}^{*}(G) \otimes S \text { holds. }
$$

This is negative answers a question of $J$. Zacharias.

\section{Preliminaries}

In this section we shall establish the basic definitions and notations for the category of coarse metric spaces. Coarse geometry is the study of the large scale properties of spaces. The notion of large scale is quantified by means of a coarse structure.

Example 2.1. [8] Let $G$ be a finitely generated group. Then the bounded coarse structure associated to any word metric on $G$ is generated by the diagonals

$$
\Delta_{g}=\{(h, h g): h \in G\}
$$


as $g$ runs over $G$.

We next recall some basic fact about uniform Roe algebra and metric property of a discrete group. Next we recall the following definitions; Let $X$ be a discrete metric space.

Definition 2.2. [8] We say that discrete metric space $X$ has bounded geometry if for all $R$ there exists $N$ in $\mathbb{N}$ such that for all $x \in X,\left|B_{R}(x)\right|<N$, where $B(x, R)=\{x \in X: d(y, x) \leq R\}$.

Definition 2.3. [8] A kernel $\phi: X \times X \longrightarrow \mathbb{C}$

- is bounded if there, exists $M>0$ such that $|\phi(s, t)|<M$ for all $s, t \in X$

- has finite propagation if there exists $R>0$ such that $\phi(s, t)=0$ if $d(s, t)>R$.

Let $B(X)$ be a set of bounded finite propagation kernels on $X \times X$. Each such $\phi$ defines a bounded operator on $\ell^{2}(X)$ via the usual formula for matrix multiplication

$$
\phi * \zeta(s)=\sum_{r \in G} \phi(s, r) \zeta(r) \text { for } \zeta \in \ell^{2}(X) .
$$

Next, we show the operator associated with a bounded kernel is bounded.

Lemma 2.4. Let $X$ be bounded geometry metric space. An operator associated with a bounded finite propagation kernel is bounded.

Proof. Let $\phi$ and $\zeta \in \ell^{2}(X)$.

Consider

$$
\begin{aligned}
\|\phi * \zeta\|_{2}^{2} & =\sum_{x \in X}|\phi * \zeta(x)|^{2} \\
& =\sum_{x \in X}\left|\sum_{y \in X} \phi(x, y) \zeta(y)\right|^{2}
\end{aligned}
$$

Given $x, \phi(x, y) \neq 0$ for $y \in B_{R}(x)$, where $R$ is the propagation of $\phi$. Consider

$$
\left|\sum_{y \in X} \phi(x, y) \zeta(y)\right| \leq \sum_{y \in X}|\phi(x, y)||\zeta(y)|
$$




$$
\begin{aligned}
& \leq \sum_{y \in X} M|\zeta(y)| \\
& \leq N_{R} M|\zeta(y)|,
\end{aligned}
$$

where, by bounded geometry $N_{R}$ is the upper bound on the number of elements in a ball $B_{R}(x)$. This is independent of $x \in X$, so

$$
\|\phi * \zeta\|_{2}^{2} \leq \sum_{x \in X} N_{R}^{2} M^{2}|\zeta(x)|^{2}=N_{R}^{2} M^{2}\|\zeta\|_{2}^{2}
$$

Therefore an operator associated with a bounded kernel is bounded.

We shall denote the finite propagation kernels on $X$ by $A^{\infty}(X)$.

Definition 2.5. The uniform Roe algebra of a metric space $X$ is the closure of $A^{\infty}(X)$ in the algebra $B\left(\ell^{2}(X)\right)$ of bounded operators on $X$.

If a discrete group $G$ is equipped with its bounded coarse structure introduced in Example 2.1 then one can associated with it uniform Roe algebra $C_{u}^{*}(G)$ by repeating the above.

An important class of $C^{*}-$ algebras arises in the study of groups. Let $G$ be a discrete group, then the characteristic function $\delta_{g}(s)$ of $s \in G$ is defined as follows [3]:

$$
\delta_{g}(s)= \begin{cases}1 & \text { if } g=s \\ 0 & \text { if } g \neq s\end{cases}
$$

If we assume that the $G$ is a discrete group then the functions $\delta_{g}$ form a basis for the Hilbert space $\ell^{2}(G)$ of square summable functions on $G$.

We denote the group ring of $G$ by $\mathbb{C}[G]$ with the multiplication and adjoint defined by

$$
\begin{gathered}
\left(\sum_{s \in G} a_{s} s\right)\left(\sum_{t \in G} a_{t} t\right)=\sum_{s, t \in G} a_{s} a_{t} s t \\
\left(\sum_{s \in G} a_{s} s\right)^{*}=\sum_{s \in G} \overline{a_{s}} s^{-1}
\end{gathered}
$$


The group ring $\mathbb{C}[G]$ consists of all finitely supported complex-valued functions on $G$, that is of all finite combinations $f=\sum_{s \in G} a_{s} s$ with complex coefficients. Denote $B\left(\ell^{2}(G)\right)$ the $C^{*}$ - algebra of all bounded linear operator on Hilbert space $\ell^{2}(G)$. We may distinguish between the left regular representation, which is induced by the left multiplication action, and the right regular representation, which is comes from the multiplication on the right. The left regular representation can be extended to an injective $*$ - homomorphism $\mathbb{C}[G] \longrightarrow B\left(\ell^{2}(G)\right)$, which we also denote by $\lambda$.

Definition 2.6. [3] The left regular representation

$$
\lambda: \mathbb{C}[G] \rightarrow B\left(\ell^{2}(G)\right)
$$

is defined by

$$
\lambda(s) \delta_{t}(r)=\delta_{t}\left(s^{-1} r\right)=\delta_{s t}(r) \text { for } s, r \in G . .
$$

The right regular representation is given by

$$
\rho(s) \delta_{t}(r)=\delta_{t}(r s)=\delta_{t s^{-1}}(r) \text { for } s, r \in G .
$$

The reduced $C^{*}$ - algebra $C_{\lambda}^{*}(G)$ of a group $G$ (which we shall assume to be discrete) arises from the study of the left regular representation $\lambda$ of the group ring $\mathbb{C}[G]$ on the Hilbert space of square-summable functions on the group.

Definition 2.7. [3] The reduced group $C^{*}$ - algebra $G$, denoted by $C_{r}^{*}(G)$ is the completion of $\mathbb{C}[G]$ in the norm given, for $c \in \mathbb{C}[G]$, by

$$
\|c\|_{\lambda}=\|\lambda(c)\|
$$

Equivalently, it is the closure of $\mathbb{C}[G]$ is identified with its image under the left regular representation. i.e.

$$
C_{\lambda}^{*}(G):=\overline{\lambda(\mathbb{C}[G])}
$$

The reduced $C^{*}$ - algebra $C_{\rho}^{*}(G)$ of a group $G$ (which we shall assume to be discrete) arises from the study of the right regular representation $\rho$ of the group ring $\mathbb{C}[G]$ on the Hilbert space of square-summable functions on the group. 
Definition 2.8. The reduced group $C^{*}-$ algebra $G$, denoted by $C_{\rho}^{*}(G)$ is the completion of $\mathbb{C}[G]$ in the norm given, for $c \in \mathbb{C}[G]$, by

$$
\|c\|_{\rho}=\|\rho(c)\|
$$

Equivalently, it is the closure of $\mathbb{C}[G]$ is identified with its image under the right regular representation.

$$
C_{\rho}^{*}(G):=\overline{\rho(\mathbb{C}[G])}
$$

A discrete group $G$ has a natural coarse structure which allows us to define the uniform Roe algebra $C_{u}^{*}(G)$. A group $G$ can be equipped with either the left or right-invariant of the metric. A choice of one of the determines whether $C_{\lambda}^{*}(G)$ or $C_{\rho}^{*}(G)$ is a sublagebra of the uniform Roe algebra $C_{u}^{*}(G)$ of $G$

We now define the invariant approximation: property (IAP)

Definition 2.9. [8] We say that $G$ has the invariant approximation prop$\operatorname{erty}(I A P)$ if

$$
C_{\lambda}^{*}(G)=C_{u}^{*}(G)^{G}
$$

We begin with a some definition of Haagerup and Kraus [5].

Definition 2.10. [1] A $C^{*}-$ algebra $A$ is nuclear if and only if it has the following completely positive approximation property (CPAP): The identity map on $A$ can be approximated in the point norm topology by finite rank completely positive contractions. This means that there exist nets of operators $T_{\alpha}: A \longrightarrow M_{n_{\alpha}}(\mathbb{C})$ and $S_{\alpha}: M_{n_{\alpha}}(\mathbb{C}) \longrightarrow A$ such that for all $a \in A$

$$
\lim _{\alpha}\left\|S_{\alpha} T_{\alpha}(a)-a\right\|=0
$$

A $C^{*}$ - algebra $A$ has the metric approximation property (MAP) of Grothendieck if and only if the identity map on $A$ can be approximated in the point-norm topology by a net of finite rank contractions.

Comparing the definitions we see that CPAP implies MAP (see for example [1]). Lance [7] has shown that $\Gamma$ is amenable if and only if its reduced $C^{*}-$ algebra $A$ has the CPAP which is equivalent to $C_{r}^{*}(\Gamma)$ being nuclear. Completely positive maps are in particular completely bounded, which suggest the following weakening of the CPAP. 
Definition 2.11. [1] A $C^{*}$-algebra $A$ is said to have the completely bounded approximation property (CBAP) if there is a positive number $C$ such that the identity map on $A$ can be approximated in the point norm topology by a net $\left\{\phi_{\alpha}\right\}$ of finite rank completely bounded maps whose completely bounded norms are bounded by $C$. This means that there exists a net of finite-rank maps $\left\{\phi_{\alpha}\right\}: A \longrightarrow A$ such that $\left\|\phi_{\alpha}\right\|_{c b} \leq C$ for some constant $C$ and $\phi_{\alpha} \longrightarrow i d_{A}$ in the point-norm topology on $A$ (i.e. $\left\|\phi_{\alpha}(x)-x\right\| \longrightarrow 0$ for all $x \in A$ ).

The infimum of all values of $C$ for which such constants exist is denoted by $\Lambda_{c b}(A)$ and is called the Cowling - Haagerup constant. We set $\Lambda_{c b}(\Gamma)=\infty$ if the locally compact group $\Gamma$ does not have the CBAP. Obviously, a nuclear $C^{*}-$ algebra has the metric approximation property. On the other hand, Haagerup [4] proved that the reduced $C^{*}-$ algebra $\mathbb{F}_{n}$ has the metric approximation property, a very remarkable result since $C_{r}^{*}\left(\mathbb{F}_{n}\right), n>2$, is not nuclear, $\mathbb{F}_{n}$ not being amenable. We have the following definition of weak amenability.

Definition 2.12. [1] An approximate identity on $G$ is a sequence $\left(\phi_{n}\right)$ of finitely supported functions such that $\phi_{n}$ uniformly converge to constant function 1 . We say that discrete $G$ is weakly amenable if there is an approximate identity $\left(\phi_{n}\right)$ such that

$$
C:=\sup \left\|M_{\phi_{\mathrm{n}}}\right\|_{c b}<\infty .
$$

We have the following important result by Haagerup [4].

Theorem 2.13. Let $G$ be a discrete group. The following are equivalent:

1. $G$ is weakly amenable,

2. $C_{r}^{*}(G)$ has the $C B A P$.

Definition 2.14. [2] We say that discrete group $G$ is amenable if and only if there is an approximate identity consisting of positive definite functions.

Lemma 2.15. An amenable discrete group is weakly amenable.

Proof. We recall that $G$ is an amenable discrete group if and only if there is an approximate identity on $G$ consisting of positive definite functions. A sequence $\left(\phi_{n}\right)$ of finitely supported functions such that $\phi_{n} \longrightarrow 1$. Then $M_{\phi_{n}}$ 
completely positive on $C_{\lambda}^{*}(G)$ and also $M_{\phi_{\mathrm{n}}}$ completely bounded and

$$
\left\|M_{\phi_{\mathrm{n}}}\right\|_{c b}=\phi(1)
$$

Thus $\Lambda_{c b}(G)=1$. Therefore $G$ has CBAP. By Theorem 2.13, $G$ is weakly amenable.

Haagerup and Kraus have provided in [5] a detailed characterisation of AP.We say that the $C^{*}$ - algebra $A$ has the strong operator approximation property (SOAP) if there is a net $T_{\alpha}$ in $A(G)$ such that $T_{\alpha} \longrightarrow i d_{A}$ in the stable point-norm topology.

If $A$ is a $C^{*}$ - algebra, and $\mathcal{H}$ is a separable infinite Hilbert space, a net $T_{\alpha}$ in $C B(A)$ is said to converge in the stable point-norm topology to $T$ in $C B(A)$ if $T_{\alpha} \otimes i d_{K(H)}(a) \longrightarrow T \otimes i d_{\mathcal{K}(\mathcal{H})}$ in norm for all $a \in A \otimes \mathcal{K}(H)$. Here $\mathcal{K}(\mathcal{H})$ denotes the ideal of compact operators on $H$.

We say that $C^{*}$ - algebra, $A$ has the operator approximation property (OAP) if there exists a net of finite - rank maps $T_{\alpha}: A \longrightarrow A$ such that $T_{\alpha} \longrightarrow i d_{A}$ in the stable point-norm topology. This means that there exists a net of finite rank linear maps

$$
T_{\alpha}: A \longrightarrow A
$$

such that for all $x \in \mathcal{K}(\mathcal{H}) \otimes_{\min } A$,

$$
\left\|I d \otimes T_{\alpha}(x)-x\right\| \longrightarrow 0 .
$$

The Fourier algebra

$$
A(G):=\left\{f: f(t)=\langle\lambda(t) \xi \mid \eta\rangle \text { for some } \xi, \eta \in \ell_{2}(G)\right\}
$$

is the space of all coefficient function of the left regular representation $\lambda$. Given $f \in A(G)$, its norm is given by

$$
\|f\|=\inf \{\|\xi\|\|\eta\|: \quad f(t)=\langle\lambda(t) \xi \mid \eta\rangle\}
$$

With this norm, $A(G)$ is a Banach algebra with the pointwise multiplication $[5]$.

A complex-valued function $\phi$ on $G$ is a multiplier for $A(G)$ if the linear map

$$
M_{\phi}(f)=\phi f
$$

sends $A(\Gamma)$ to $A(\Gamma)$. If the map $M_{\phi}$ is completely bounded on $A(G)$, we call $\phi$ a completely bounded multiplier of $A(G)$. The set of multipliers of $A(G)$ is 
denoted by $M_{0} A(G)$. If $\phi \in A(G)$ then $\phi$ is a bounded continuous function and $M_{\phi}$ is a bounded operator on the space $A(G)$.

The discrete group $G$ has the approximation property (AP) if there is a net $\left\{\phi_{\alpha}\right\}$ in $A(G)$ such that $M_{\phi_{\alpha}} \longrightarrow i d_{A(G)}$ in the stable point-norm topology on $A(G)$.

We have the following important result from Haagerup and Kraus [5]:

Theorem 2.16. Let $G$ be a discrete group. Then the following are equivalent:

1. G has the AP,

2. $C_{r}^{*}(G)$ has the operator approximation property (OAP),

3. $C_{r}^{*}(G)$ has the strong operator approximation property (strong OAP).

Example 2.17. The following groups have AP [5]. This implies that these groups have the OAP, and thus also SOAP:

- $S L(2, \mathbb{Z})[5]$

- $\mathbb{Z}^{2} \rtimes S L(2, \mathbb{Z})[5]$

In this section we will give definition of the strong invariant approximation property. Let $S \subseteq B(\mathcal{H})$ be a closed subspace.

Next, we define the set of fixed points of $C_{u}^{*}(G, S)^{G}$ :

Definition 2.18. We define

$$
C_{u}^{*}(G, S)^{G}=\left\{T \in C_{u}^{*}(G, S) ; A d\left(\rho_{t} \otimes i d\right) T=T \text { for all } t \in G\right\} .
$$

We now define Joachim Zacharias's IAP with coefficients (SIAP):

Definition 2.19. [9] We say that a discrete group $G$ has the strong invariant translation approximation property (SIAP) if for any closed subspace $S$ of the compact operators $\mathcal{K}\left(\right.$ on $\left.\ell^{2}(\mathbb{N})\right)$. We have an isomorphism

$$
C_{u}^{*}(G, S)^{G}=C_{\lambda}^{*}(G) \otimes S \text { holds. }
$$




\section{Analytic Properties of Strong IAP}

In this section, we show some of the analytic properties of the strong invariant approximation property for discrete exact groups.

Theorem 3.1. [9]For a discrete exact group $G$ the following are equivalent.

1. G has the AP.

2. $C_{r}^{*}(G)$ has the $O A P$.

3. G has SIAP (Zacharias's IAP with coefficients)

The above Theorem means for a discrete exact group the following properties are actually equivalent:

$$
A P \Longleftrightarrow O A P \Longleftrightarrow S I A P .
$$

Theorem 3.2. For a discrete exact group $G$. $G$ has the SIAP if for any closed subspace $S \subseteq B(\mathcal{H})$ the equality

$$
C_{u}^{*}(G, S)^{G}=C_{\lambda}^{*}(G) \otimes S \text { holds. }
$$

Proof. Since $G$ has IAP, so

$$
C_{u}^{*}(G)^{G}=C_{\lambda}^{*}(G)
$$

and therefore

$$
C_{u}^{*}(G, S)^{G}=C_{u}^{*}(G)^{G} \otimes S
$$

for any closed subspace $S \subseteq B(\mathcal{H})$.

First we recall the following definitions.

Definition 3.3. [2] We define

$$
S L_{2}(\mathbb{Z})=\left\{\left(\begin{array}{ll}
a & b \\
c & d
\end{array}\right): a, b, c, d \in \mathbb{Z} \text { and } a d-b c=1\right\} .
$$

Remark 3.4. For discrete groups we have the following implications:

Amenability $\Longrightarrow$ weak amenability $\Longrightarrow \mathrm{AP} \Longrightarrow$ exactness. 
The first implication is explained in Lemma 2.15. The first implication is not an equivalence: the non-abelian free groups are weakly amenable, but they are not amenable. The second implication is proved by Haagerup and Kraus showed in [5] and also this implication is not an equivalence: a counter-example is given by $\mathbb{Z}^{2} \rtimes S L(2, \mathbb{Z})$; this group has the AP [5]. But it was proved in [5] that it is not weakly amenable. The third implication is not an equivalence: Haagerup and Kraus showed in [5] that $S L_{2}(\mathbb{Z})$ is an exact group without AP.

We show the following Proposition:

Proposition 3.5. If $G=S L_{2}(\mathbb{Z})$. Then there exist any Hilbert space $B(\mathbb{H})$ and closed subspace $S \subseteq H$ such that property if and only if

$$
C_{u}^{*}(G, S)^{G} \neq C_{\lambda}^{*}(G) \otimes S \text { holds. }
$$

Proof. Since $G$ has not AP [5]. But Zacharia's show that $G$ has AP if and only if $G$ has SIAP. Therefore $G$ has not SIAP. This mean

$$
C_{u}^{*}(G, S)^{G} \neq C_{\lambda}^{*}(G) \otimes S \text { holds. }
$$

\section{References}

[1] J. Brodzki, G.A. Niblo, Approximation properties for discrete groups, In: $C^{*}$-Algebras and Elliptic Theory, Trends Math, Basel (2006), 23-35.

[2] P. Brown, N.G. Ozawa, $C^{*}$ and Finite Dimensional Approximations, Graduate Studies in Mathematics, American Mathematical Soc., 88 (2008).

[3] K.R. Davidson, $C^{*}$-algebra by Example, Field Institute Mono-Graphs, American Mathematical Soc., Providence, RI, 6 (1986).

[4] U. Haagerup, An example of a non nuclear $C^{*}$-algebra, which has the metric approximation property, Inventiones Mathematicae, 50, No. 3 (1978), 279-293.

[5] U. Haagerup, J. Kraus, Approximation properties for group $C^{*}$-algebras and group Von Neumann algebras, Inventiones Mathematicae, 344, No. 2 (1994), 667-699. 
[6] K. Kannan, On Approximation Properties of Group $C^{*}$-Algebras, University of Southampton, School of Mathematics, Doctoral Thesis, No. 2 (2011), 116pp.

[7] E.C. Lance, On nuclear $C^{*}$ - algebras, Journal of Functional Analysis, 12 (1973), 157-176.

[8] J. Roe, Lectures on coarse geometry, American Mathematical Society, 31 (2003).

[9] J. Zacharias, On the invariant translation approximation property for discrete groups, Proc. Amer. Math. Soc., 134, No. 7 (2006), 1909-1916. 